\title{
INVESTIGATION OF FLAME STRUCTURE AND SOOT FORMATION IN A SINGLE SECTOR MODEL COMBUSTOR USING EXPERIMENTS AND NUMERICAL SIMULATIONS BASED ON THE LES/CMC APPROACH
}

\author{
Andrea Giusti* \\ Epaminondas Mastorakos \\ Department of Engineering \\ University of Cambridge \\ Trumpington Street, CB2 1PZ, \\ Cambridge, UK \\ Email: ag813@cam.ac.uk
}

\author{
Christoph Hassa \\ Johannes Heinze \\ Eggert Magens
}

\author{
German Aerospace Center (DLR) \\ Linder Hoehe, Cologne, Germany
}

\author{
Marco Zedda \\ Combustion Aerothermal Methods \\ Rolls-Royce plc. \\ PO Box 31, Derby DE24 8BJ, UK
}

\begin{abstract}
In this work a single sector lean burn model combustor operating in pilot only mode has been investigated using both experiments and computations with the main objective of analyzing the flame structure and soot formation at conditions relevant to aero-engine applications. Numerical simulations were performed using the Large Eddy Simulation (LES) approach and the Conditional Moment Closure (CMC) combustion model with detailed chemistry and a two-equation model for soot. The CMC model is based on the time-resolved solution of the local flame structure and allows to directly take into account the phenomena associated to molecular mixing and turbulent transport which are of great importance for the prediction of emissions. The rig investigated in this work, called Big Optical Single Sector (BOSS) rig, allows to test real scale lean burn injectors. Experiments, performed at elevated pressure and temperature, corresponding to engine conditions at part load, include OH-PLIF and PDA and have been complemented with new LII measurements for soot location. The wide range of measurements available allows a comprehensive analysis of the primary combustion region and can be exploited to further assess and validate the LES/CMC approach to capture the flame behaviour at engine conditions. It is shown that the LES/CMC approach is able to predict the main characteristics of the flame with a good agreement with the experiment in terms of flame shape, spray characteristics and soot location. Finite-rate chemistry effects appear to be very important in the region very close to the injection location leading to the lift-off of the flame. Low levels of soot are observed immediately downstream of the injector exit, where a high amount of vaporized fuel is still present. Further downstream, the fuel vapour disappears quite quickly and an extended region characterised by the presence of pyrolysis products and soot precursors is observed. The strong production of soot precursors together with high soot surface growth rates lead to high values of soot volume fraction in locations consistent with the experiment. Soot oxidation is also very important in the downstream region resulting in a decrease of the soot level at the combustor exit. The results show a very promising capability of the LES/CMC approach to capture the main characteristics of the flame, soot formation and location at engine relevant conditions. More advanced soot models will be considered in future work in order to improve the quantitative prediction of the soot level.
\end{abstract}

\section{INTRODUCTION}

Due to the increasingly stringent regulations for reduction of the environmental impact of aviation, lean burn concepts are being developed by aero-engine manufacturers. Lean burn designs for aero-engine applications rely on Lean Direct Injection (LDI) and piloted fuel injectors. At high power conditions the fuel injectors are operated in pilot and main mode in order to produce a lean flame, giving low levels of $\mathrm{NO}_{\mathrm{x}}$ and soot. At mid- to low-power conditions, however, the injectors are operated in pilot only mode. The resulting flame shows features typically associated with the diffusion combustion regime, which may give rise to non-negligible levels of soot. Pilot only soot emissions are the main factor defining the operating point at which the main fuel circuit is switched on, together with

*Address all the correspondence to this author. 
considerations about pilot and main combustion efficiency. So, at the design stage, it is critically important to be able to characterise the flame structure and soot emissions at the pilot only condition.

The formation and evolution of pollutant species in aero-engines involve many physical processes, ranging from chemistry to turbulence-flame interaction, which should be properly addressed in order to have a reliable prediction of emissions. In particular, a reliable prediction of soot formation requires an accurate description of the finite-rate chemistry and turbulent transport effects of large and small scales [1]. The presence of an evaporating spray makes the physical phenomena even more complex due to the wide range of scales involved [2] and its direct effect on the mixing field [3] and flame structure [4]. In the context of CFD simulations, it is therefore crucial to use a computational approach able to properly address all these multi-scale interactions as well as to develop advanced soot models able to describe the phenomena leading to the formation of soot particles and their subsequent evolution involving surface growth, oxidation and coagulation. In this work, the Large Eddy Simulation (LES) approach together with the single-conditioned Conditional Moment Closure (CMC) combustion model and a two-equation model for soot have been used. The capability of the LES/CMC approach to capture finite-rate turbulence-chemistry interactions has already been extensively validated in lab-scale flames for both gaseous and spray flames [5-10]. In particular, simulations of swirling non-premixed methane flame have shown that LES/CMC is able to give a reliable prediction of local extinction and its evolution into a global blow-off with a deviation in the prediction of the experimental blow-off curve within $25 \%$ of the experimental blow-off velocity $[7,11]$. The capability of the LES/CMC approach to quantitatively capture local extinctions has also been shown in the context of spray flames [9]. Interaction of the reacting field with the evaporation can induce high sub-grid scale gradients, increasing the amount of local extinction $[9,12]$ with a strong impact on the evolution of the global blow-off [13]. As far as the prediction of soot is concerned, recent work $[14,15]$ reported a promising capability of the LES/CMC approach to predict the formation and location of the soot using relatively simple soot models.

The main objective of this work is to analyze the flame structure and soot formation in the primary combustion region of a model combustor operated in pilot only mode, using both experiments and numerical simulations. Although the LES/CMC approach has been applied so far to the simulation of many lab-scale experiments, to the authors' knowledge a LES/CMC simulation of a practical injector at engine conditions has not been attempted before. Therefore, comparisons between numerical results and experimental measurements discussed in the present work also allow further assessment and validation of the capability of the LES/CMC approach to capture the flame characteristics at conditions relevant to aero-engine applications and with real injector geometries.

The paper is structured as follows. The model combustor investigated in this work is first introduced, followed by a brief description of the experimental techniques used for the visualization and the measure of relevant quantities in the primary combustion region and the numerical methods used for the computation. Results are then presented and discussed with a focus on the flame structure and soot location in the primary region of the combustor. Conclusions and recommendations for future work close the paper.

\section{THE INVESTIGATED COMBUSTOR}

The rig investigated in this work [16], called Big Optical Single Sector (BOSS), is a single sector model combustor reproducing the main characteristics of a real engine. The rig allows operation of original engine-size lean burners with pressures up to 20 bar and provides a wide optical access to the primary zone of the combustor enabling the use of planar laser-based spectroscopic techniques. Figure 1 shows the main characteristics and components of the test section [17]. The combustion chamber comprises a square section enclosure with dimensions $140 \times 140 \mathrm{~mm}$ immediately downstream of the injector exit. The optical segment has access from three sides (a) and extends from the injector exit $40 \mathrm{~mm}$ downstream. The bottom side (b) holds a hydrogen torch igniter. The inner surface of all the four sides of the optical segment is protected by a starter film of $1 \mathrm{~mm}$ thickness [16], with adjustable mass flow and temperature. In the experiments performed in this work, the temperature of the film was set equal to the temperature, $T_{30}$, of the air flowing through the injector with a mass flow rate of about $30 \%$ of the mass flow through the injector. The downstream part of the rig is characterised by an effusion-cooled square transition piece (c) and an effusion-cooled converging section (e). The cooling air used in the downstream part of the rig is at ambient temperature with controllable mass flow rate. A feed through in the region of the secondary zone of pressure vessel and flame tube (f) provides access to the combustor for an optical probe. The effusion-cooled transition piece (c) can be exchanged with the optical segment (a) to shift the optical accessible region further downstream. Alternatively, larger windows can be used to observe the entire region (0-90 mm downstream of the injector exit) covered by the pressure window (d). The overall length of the combustor is about $250 \mathrm{~mm}$ (further details regarding the geometry and cooling configuration can be found in Ref. [16]). The rig is operated with kerosene (Jet-A1) as fuel. The injector (g) investigated in the present work is based on a two-stage configuration with the main and pilot fuel injection achieved through concentric airblast atomizers (more details on the injector configuration can be found in Ref. [18]). The outer airblast atomizer represents the main fuel injection with the pilot injector located at the center. High velocity swirling flows allow a fine primary atomization and the formation of a recirculation region inside the combustion chamber for the stabilisation of the flame. The pilot atomizing edge is located few mm upstream of the injector exit plane, where the optical access is 
provided. The condition investigated in this work corresponds to a part load condition where the injector is operated in pilot only mode. The operating pressure is the one of a low-power pilot only condition with an overall air-to-fuel ratio (including all the cooling flows) of about 125. Experiments include PDA (Phase Doppler Anemometry) measurements [17] for spray characterisation, and OH-PLIF (Planar Laser-Induced Fluorescence of $\mathrm{OH}$ ) and have been complemented with new LII (Laser-Induced Incandescence) measurements for soot location. The evaluation of these techniques allows to follow the general features of the combustion process from the liquid phase through evaporation, reaction and finally soot formation with high spatial resolution. Further details regarding the characteristics of the rig can be found in Refs. [16,17].

\section{METHODS}

In this section the measurement techniques and experimental setup together with the numerical approach and models used in the computations are briefly summarized. For further details regarding both the experimental and numerical methods, the reader should consult the specific references indicated.

\section{Experimental methods}

The following planar optical measurement techniques were applied for the characterisation of the combustor at DLR: (i) PDA (measurement of the droplet diameters, see Ref. [17] for details), (ii) OH-PLIF (high temperature regions, mixing, temperature distribution) and (iii) LII (quantitative soot volume fraction). The hardware for a combination of several spectroscopic techniques was accommodated in a single container placed near the test section. The experimental setup used in the experiments is shown in Fig. 2. Three pulsed laser systems generate radiation with different wavelengths for LII or soot absorption (L3, $1064 \mathrm{~nm}$ ), Mie scattering of liquid fuel or soot absorption (L2, $532 \mathrm{~nm}$ ), and UV for OH-PLIF ( $283 \mathrm{~nm}, \mathrm{~L} 1)$. The beams are superimposed, formed into light sheets and focused into the combustor. A small fraction of the laser light is split off before entering and after leaving the combustor. It is guided into cells filled with a fluorescing dye solution for visible and UV light, and under grazing incidence onto a white surface for near infrared $1064 \mathrm{~nm}$ radiation. Light emitted from these "references" is recorded by two cameras which measure both the power distribution across the laser light sheets, as well as the integral absorption across the combustor. The beam profiler controls the correct overlap of the $532 \mathrm{~nm}$ and the $1064 \mathrm{~nm}$ beams. This is necessary because the absorption of the $532 \mathrm{~nm}$ beam is used to calibrate the LII signal generated by the $1064 \mathrm{~nm}$ beam. Therefore, it has to be ensured that both beams travel through identical regions of the flame. Furthermore, thermal drifts in the test cell may cause a beam shift over a test duration of several hours; the beam profiler helps to compensate for such deviations accordingly, using remotely controlled mirrors. By careful correction procedures taking into account the simultaneously measured absorption, quantitativeness of soot concentration up to a medium soot level can be achieved. Planar signals are recorded by camera 1 (LII, Mie scattering or chemiluminescence, depending on the spectral filtering controlled by the filter wheel) and camera 2 (OH-PLIF).

The method of single shot temperature measurement by PLIF on OH has been described in [19]. It is based on a quantitative measurement of the $\mathrm{OH}$ concentration, which is achieved using corrections from careful absorption measurements. It further assumes the concentration of $\mathrm{OH}$ to be in partial equilibrium on the lean side of stoichiometry, which further limits its use beginning in some distance to the flame front. Given these limitations of the single line temperature measurement, the technique is not well suited for the temperature measurement in a rich zone. However, it is the high signal strength of the chosen transition that enables operation in an adverse environment and justifies its use here. The experimental situation is characterised by a comparatively long beam path in the hot environment at elevated pressure, interference from soot and a multitude of window surfaces to be crossed. All these factors demand sufficient signal for simultaneous 2D measurements, the subsequent normalizations and corrections additionally requiring reasonable signal resolution for a useful application.

A schematic of the streamwise cross section of the combustor with the observation windows used in both OH-PLIF and LII measurements is shown in Fig. 3, to illustrate the orientation of the planar measurements with respect to the combustor boundaries and the fuel injector. The location of PDA measurements considered in the following for comparisons with the spray Sauter Mean Diameter (SMD) predicted by the CFD is also shown. An error estimation for the temperatures deduced from OH-PLIF measurements was conducted. Following Heinze et al. [19], the various measurement uncertainties add up to an error of $\pm 20 \%$ in OH concentration. Because of the favourable nonlinear relation between $\mathrm{OH}$ and $\mathrm{T}$, this results in an error of less than 3\% for temperatures around 1800-2200 K in the exhaust of lean flames. However, under non-equilibrium conditions within the flame front itself, the superequilibrium $\mathrm{OH}$ concentration (about 1.5-2 times higher) leads to an overestimation of the temperature of 100-200 K. In pressurized flames the relaxation of $\mathrm{OH}$ concentration to equilibrium takes place within parts of $\mathrm{ms}$ or in a (few) $\mathrm{mm}$. As far as LII measurements for soot are concerned, the condition investigated in this work is characterized by a relatively low soot concentration, with moderate implications of absorption on the signal. The dominant error is introduced by the luminous flame background producing some noise. Hence the conditions for a 
calibration by absorption are rather favourable with an accuracy of 5\% to $10 \%$ resulting in an overall error in the measurement lower than $20 \%$. For PDA measurements, the error for SMD is about $3 \%$ in undisturbed flow. However, in a reacting condition, considering the temperature influence on refractive index and rejection of reflection, the accuracy can be estimated to be within 10\% (see also Ref. [17] for a more detailed description of PDA experiments).

\section{Numerical methods}

Numerical simulations were performed at University of Cambridge using the LES approach and CMC combustion model [20,21] with mixture fraction as conditioning variable. The spray was modelled using a two-way coupled Eulerian-Lagrangian approach for dilute sprays. The filtered continuity and momentum equations together with transport equations for the mixture fraction, $\widetilde{\xi}$, and its sub-grid scale variance, $\widetilde{\xi^{\prime \prime 2}}$, and the spray evolution were solved using the unstructured finite volume code PRECISE-UNS [22]. Spray source terms were also included in the sub-grid scale mixture fraction variance equation following the models discussed in [9] and references therein. This allows to include the spray effects on the sub-grid scale gradients, which can have an important impact on the overall sub-grid scale variance, as pointed out in recent work [3,9].

CMC modelling. The conditionally filtered equations for species mass fractions and enthalpy were solved by means of an unstructured in-house CMC solver [6,7]. The CMC equation for a generic conditionally filtered reacting scalar $\widetilde{Y_{\alpha} \mid \eta}=Q_{\alpha}(\eta$ is the sample space variable), can be written as [23]:

$$
\frac{\partial Q_{\alpha}}{\partial t}+\underbrace{\widetilde{u_{i} \mid \eta} \frac{\partial Q_{\alpha}}{\partial x_{i}}}_{T 1}=\underbrace{\widetilde{N \mid \eta} \frac{\partial^{2} Q_{\alpha}}{\partial \eta^{2}}}_{T 2}+\underbrace{\widetilde{\omega_{\alpha} \mid \eta}}_{T 3}+\underbrace{e_{\alpha}}_{T 4}+\underbrace{S_{\alpha}(\widetilde{\Pi \mid \eta})}_{T 5}
$$

where $e_{\alpha}$ represents the sub-grid scale contributions and was modelled with a gradient assumption neglecting the sub-grid joint fluctuation of species mass fraction and droplet evaporation rate $[8,23] . \widetilde{u_{i} \mid \eta}$ is the conditionally filtered velocity, modelled as $\widetilde{u_{i} \mid \eta}=\widetilde{u_{i}}$ whereas $\widehat{N \mid \eta}$ is the conditionally filtered scalar dissipation rate which was modelled using the Amplitude Mapping Closure model [24]: $\widetilde{N \mid \eta}=N_{0} G(\eta)$, with $G(\eta)=\exp \left(-2\left[\operatorname{erf}^{-1}(2 \eta-1)\right]^{2}\right)$ and $N_{0}=\widetilde{N} / \int_{0}^{1} \widetilde{P}(\eta) G(\eta) d \eta$. $\widetilde{P}(\eta)$ is the mixture fraction Filtered probability Density Function (FDF) assumed to have a presumed $\beta$-function shape and calculated from $\widetilde{\xi}$ and $\widetilde{\xi^{\prime \prime 2}}$. The filtered scalar dissipation rate $\widetilde{N}$ is computed starting from the LES solution of the gas-phase and it consists of contributions from both resolved and sub-grid fields:

$$
\widetilde{N}=\underbrace{D \frac{\partial \widetilde{\xi}}{\partial x_{i}} \frac{\partial \widetilde{\xi}}{\partial x_{i}}}_{\text {resolved }}+\underbrace{\frac{1}{2} C_{N} \frac{\mu_{s g s}}{\bar{\rho} \Delta^{2}} \widetilde{\xi \prime 2}}_{\text {sub-grid scale }}
$$

where $D$ is the molecular diffusivity, $\mu_{s g s}$ is the sub-grid scale viscosity (constant Smagorinsky model was used for the sub-grid scale stress tensor), $\rho$ is the density, $\Delta$ is the LES filter size, and $C_{N}$ is a model constant taken equal to 42.0 [25]. The term $\widetilde{\omega_{\alpha} \mid \eta}$ in Eq. 1 is the conditionally filtered reaction rate, closed with a first order approximation, whereas $S_{\alpha}(\widetilde{\Pi \mid \eta})$ represents terms related to the contribution due to spray evaporation, closed using the models discussed in [23]. A similar equation, without chemical source term, is also solved for the conditionally filtered enthalpy. The coupling between the LES and CMC solvers is achieved through density and temperature with the CMC solver that receives flow field quantities from the LES (including spray source terms) and gives back to the LES solver the unconditional temperature and density (unconditional values are obtained from the respective conditional values through the integration over the FDF).

Chemistry and soot modelling. As observed in previous work, in order to properly capture the soot evolution it is important to have an accurate model for the turbulence-chemistry interactions [1]. The level of soot at the exit of a combustor is typically the result of generation and oxidation processes, which are strongly influenced by the local fluctuations of the soot precursor, turbulence-related 
strain and combustion [26]. Turbulent transport and mixing at both the resolved and sub-grid scales are crucial phenomena for the prediction of soot evolution [27]. It is therefore advantageous to use the CMC framework where the unsteady evolution of the local flame structure is solved, including transport in physical space due to both resolved and sub-grid fluctuations (terms T1 and T4 in Eq. 1), diffusion in mixture fraction space (micro-mixing, T2) and effects of spray evaporation (term T5 in Eq. 1).

Furthermore, in order to have access to the soot precursors, detailed chemical mechanisms should be used. In this work, kerosene was modelled as a single-component surrogate with dodecane as the main species, and a detailed chemical mechanism for dodecane with 38 species (based on the mechanism of Ref. [28] and specifically calibrated for kerosene combustion) was used. In order to predict soot, the CMC model was coupled with a two-equation model for soot [29], following the strategy discussed in previous work [15]. This model is based on the transport of two additional quantities, namely the soot mass fraction and the soot number density. Conditionally filtered equations [14] are solved for both these quantities. The soot chemistry is based on the acetylene $\left(\mathrm{C}_{2} \mathrm{H}_{2}\right)$ as a precursor for soot nucleation and includes soot surface growth, oxidation (by both $\mathrm{O}_{2}$ and $\mathrm{OH}$ ) and coagulation [15, 29]. Although simple, this model coupled with CMC has already been demonstrated to be able to give reasonable predictions of soot formation and location in high-pressure flames $[14,15]$. Taking advantage of the soot measurements performed in the primary combustion region of the model combustor investigated in this work, here the capability of the LES/CMC coupled with the two-equation soot model to capture soot in an aero-engine configuration is further assessed and validated. The coupling of the CMC solver with more advanced soot models (e.g. $[1,30])$ will be addressed in future work.

Spray modelling. Numerical parcels were injected at the atomizing edge of the pilot injector, as schematically shown in Fig. 3. Droplet diameters were sampled from a Rosin-Rammler distribution with SMD computed from the Sattelmayer correlation [31], whereas the magnitude of the injection velocity was assumed constant for all the parcels. In order to reproduce the typical dispersion observed in airblast atomization, droplets were injected with a random injection angle sampled from a Gaussian distribution [13] with standard deviation equal to $10^{\circ}$ and mean direction aligned to the injector axis. Droplet evaporation was modelled with the Abramzon and Sirignano model [32] with an infinite conductivity assumption for the liquid. The secondary breakup of liquid droplets was accounted for by means of the model proposed by Schmehl et al. [33].

Numerical setup and boundary conditions. The computational domain, shown in Fig. 4, reproduces the geometry of the experimental rig, including the plenum upstream of the injector. Following a common practice [24], CMC equations were solved in a mesh coarser than the one used for the flow field equations. A hexa-dominant mesh of about 14 million cells with a grid size $\Delta \approx 0.8 \mathrm{~mm}$ in the whole flame region was used for the LES, whereas the CMC equations were solved in a polyhedral mesh of about 10,000 cells refined in the combustor primary region. This mesh was obtained through agglomeration of LES cells which allows to directly conserve the mass fluxes computed at the LES resolution [6]. The transfer of quantities from the LES resolution to the CMC resolution was achieved through a FDF-weighted average [24]. A grid sensitivity analysis was also performed in preliminary computations, and the resolution of the LES grid used in this work was found sufficient to properly capture all the features of the flow field and to resolve more than $85 \%$ of the turbulence kinetic energy in the entire flame region. A uniform velocity profile (pure air composition) was imposed at the inlet of the upstream plenum and at the boundary representing the window cooling slot (see also Fig. 4). No turbulent fluctuations were imposed at the inlet boundaries being the level of turbulence in the reacting region mainly dominated by the velocity fluctuations generated by the shear flows due to the strong swirling structures. Uniformly distributed point source approach was used for the modelling of the effusion cooling system and heatshield cooling. No-slip adiabatic condition was used for all the other solid boundaries and an outlet condition was imposed at the exit of the combustor. The mixture fraction space was discretized by means of 51 nodes clustered around the stoichiometric mixture fraction $\left(\eta_{s t} \approx 0.063\right)$. Pure air at $T_{30}$ was imposed at $\eta=0$ whereas pure vaporised fuel at saturation conditions was imposed at $\eta=1$. For the CMC equations, zero gradient condition was used at the outlet and solid walls and the inert mixing solution was imposed at the air inlets. Effusion cooling was treated as a mass source at the inert mixing condition. Differently from the experiment, the temperature of the effusion cooling air was assumed equal to $T_{30}$ instead of ambient temperature to avoid special treatment of the boundary condition in mixture fraction space at $\eta=0$. The effusion cooling plates are located in the downstream part of the combustor, sufficiently far from the measurement area, and, as observed in preliminary computations, the temperature of these cooling flows, relatively close to $T_{30}$, has a negligible impact on the flame structure in the primary region. Second order accurate numerical schemes, both in space and time, were used for the LES. Operator splitting technique [7,23] was used for the solution of the CMC equations. Transport in physical space was solved first followed by diffusion in mixture fraction space with spray source terms, and the chemical source term integration as the last step. First order approximation in time was adopted, with upwind scheme for the convection terms and second order schemes for diffusion terms. Integration of the chemistry was performed using the VODPK solver. A time step equal to $1 \mu \mathrm{s}$ was used for both the CMC and LES solvers allowing a CFL number lower than unity in the 
whole flame region.

\section{RESULTS AND DISCUSSION}

In the following, experimental and numerical results will be discussed with the main aim of investigating the flame characteristics and soot formation, and assess the capability of the LES/CMC approach to capture the behaviour of the flame at conditions relevant to gas-turbine applications.

\section{Mean flame structure}

Figure 5 shows the mean temperature field in the primary reacting region deduced from OH-PLIF measurements and the mean temperature from the simulation. Apart from the asymmetry of the temperature distribution, which is rendered by both experiment and calculation, three regions can be discerned in both plots. A low temperature region in the middle behind the injector visualizing the mean flame lift, a region of medium temperatures around the first and towards the main flow and a high temperature region on the right. The reaction starts with the burner air and triggers $\mathrm{OH}^{*}$ chemiluminescence up to a radius separating the two distinct red patches of the measured temperature, where the temperature predicted by the CFD is significantly lower. Up to that radius the coexistence of chemiluminescencent $\mathrm{OH}^{*}$ (observed in a very similar operating condition - not shown here) and $\mathrm{OH}$ suggests the higher probability of superequilibrium which causes an overestimation of the temperature from the measurements. Hence the difference between measurement and calculation in the central region is smaller in reality. Outside of the mean $\mathrm{OH}^{*}$ region, the burner oxygen is scarce and combustion progresses mainly on the rich side of stoichiometry. Here the nonlinearity of the OH-PLIF signal gives a too low temperature. Although the quantitative accuracy of the measurement is low, it supports the calculated topology. The overall agreement can be considered satisfactory and this demonstrates the capability of the numerical approach used in this work to capture the mean characteristics of the flame.

Figure 6 shows comparisons between the experiment and the numerical simulation in terms of Sauter Mean Diameter (SMD) of the spray at a location downstream of the injector exit. The SMD used for injection in the LES simulation has been used as reference value. The SMD predicted by the numerical simulation is in relatively good agreement with the experiment therefore demonstrating the overall reliability of the approach and boundary conditions in reproducing the main characteristics of the spray downstream of the injector exit. Small discrepancies arise very close to the injector axis where no droplets are detected in the simulation. This might be due to the lower dispersion of the droplets (also seen through comparisons with Mie scattering images, not shown here). Several factors may contribute to the lower dispersion of the spray, ranging from the injection boundary conditions to a possible underestimation of the level of fluctuation of the flow downstream of the atomizing edge. As will be discussed in the following, the region close to the injector exit is characterised by local extinction with a strong coupling with the evaporating spray and therefore the aerodynamics in such region is the result of very complex interactions.

\section{Instantaneous flame structure}

The instantaneous flame shape predicted by the CFD is revealed in Fig. 7 through an isosurface of the stoichiometric mixture fraction coloured with temperature. The green surface indicates the atomizing edge of the pilot injector. The external part of the flame has a conical shape mainly determined by the aerodynamics of the outer swirler. The stoichiometric mixture fraction surface is almost attached to the injection location, however the flame appears lifted-off, as revealed by the low temperature and negligible amount of $\mathrm{OH}$ (not shown here) close to the burner exit. The phenomena leading to the lift-off will be further analyzed in the following. The high level of turbulence induced by the strong swirling flows determines a corrugation of the flame. Gradients of mixture fraction induce a non-negligible scalar dissipation rate making finite-rate chemistry effects generally important.

The flame structure is analyzed in more detail in Fig. 8 where selected flow field quantities in a streamwise cross section of the combustor are shown. Small droplet diameters, typical of the airblast injector configuration investigated in this work, cause a fast evaporation of the fuel giving high level of mixture fraction, $\widetilde{\xi}$, close to the injection location. The strong evaporation also induces quite high fluctuations of the mixture fraction at the sub-grid scale level as indicated by the high value of sub-grid scale mixture fraction variance, $\widetilde{\xi^{\prime \prime 2}}$, in this region. This phenomenon, already observed in the simulation of lab-scale flames $[9,34]$, is here even more pronounced due to the strong evaporation of the fuel.

The scalar dissipation rate in the region close to the injector exit is in general very high with the sub-grid scale contribution, $\widetilde{N}_{s g s}$, prevailing over the resolved part, $\widetilde{N}_{\text {res }}$. This is related to the high value of sub-grid scale mixture fraction variance being the model used for the scalar dissipation rate based on the typical linear-relaxation assumption which directly relates the sub-grid scale contribution to 
the sub-grid variance itself. It should be noted that, when an evaporating spray is investigated, the linear-relaxation assumption might not be fully representative of the actual behaviour of the scalar dissipation [9], since the rate of dissipation is affected not only by the cascade from the resolved scales but also from the fluctuations added at the small scales (this phenomenon is similar to what observed for a progress variable-based computation where the reaction sources affect the progress variable behaviour at the small scales [35]). The modelling of the effect of sources at the sub-grid scale level on the scalar dissipation rate should be addressed in future work.

The high value of scalar dissipation rate makes finite-rate chemistry effects very important. Close to the injector exit, in the regions lying along the stoichiometric mixture fraction, low levels of temperature and negligible $\mathrm{OH}$ mass fraction are observed denoting local extinction in this region. Therefore, the LES/CMC solution suggests that the lift-off of the flame in this configuration is due to the localized extinction induced by the high scalar dissipation rate with spray effects that play an important role in the overall value of the filtered scalar dissipation rate.

Figure 9 shows the mass fraction of selected species in a streamwise cross-section of the combustor. The region close to the injector exit is characterised by high values of fuel mass fraction. Moving downstream, the pyrolysis of the fuel leads to the formation of intermediate species including acetylene, which is the intermediate pyrolysis product considered as soot precursor in the two-equation soot model used in this work. Regions with intense pyrolysis are characterized by a negative heat release rate (HRR), indicated by the red isoline in Fig. 8. The $\mathrm{OH}$ is generally confined in a thin region around the stoichiometric value, a typical characteristic of diffusion flames, whereas the formaldehyde mainly appears on the rich side of the flame and in regions characterised by a high scalar dissipation rate. It is also interesting to analyze the behaviour of the $\mathrm{OH} \times \mathrm{CH}_{2} \mathrm{O}$ product. By comparison with the heat release rate field in Fig. 8, it is possible to note that this quantity is able to give a good representation of the reacting region in accordance with recent investigations performed with kerosene laminar counterflow flames [36].

\section{Soot formation}

Figure 9 also shows the soot mass fraction and soot number density. High values of soot mass fraction are generally observed far from the injector exit, where the mass fraction of $\mathrm{C}_{2} \mathrm{H}_{2}$ is high, whereas close to the injection location the level of soot is lower. On the contrary, the levels of soot number density appear more uniform in the domain due to the competing mechanisms of nucleation and coagulation of soot particles. Peaks of soot are observed in the rich region of the flame, between the stoichiometric mixture fraction and the peaks of $\mathrm{C}_{2} \mathrm{H}_{2}$, consistently with recent work performed with the same approach [15]. To give more insight into the soot formation, the soot mass fraction source terms due to nucleation, surface growth and oxidation are shown in Fig. 10. The contribution due to nucleation is in general smaller compared to the other two terms showing that the level of soot in the downstream part of the flame is mainly related to the surface growth. In this region the soot oxidation is also important contributing to the consumption of the soot.

Figure 11 compares the experimental measurements of mean soot volume fraction with the values predicted by the numerical simulation. Numerical simulations are in good agreement with the experiment in terms of soot location. The low value of soot volume fraction close to the injector exit and its increase in the downstream direction as well as the peak location are quite well predicted. The appearance of non-negligible amounts of soot along the combustor axis in the downstream region is also captured by the simulation. As far as the absolute level of soot is concerned, the simulations generally overpredict the experimental soot level. This can be attributed to the simple form of the soot model used in this work where only the acetylene is used as precursor for the soot formation. Nevertheless, the good agreement in terms of soot location shows a promising degree of maturity of the LES/CMC approach to capture emissions at conditions of industrial relevance. Future work should look at the coupling of the CMC model with more advanced soot models (e.g. sectional models) which, together with a more detailed chemistry, may give more accurate quantitative prediction of the soot level.

\section{CONCLUSIONS}

The flame structure and soot formation in a single sector model combustor have been investigated using both experiments and numerical simulations. Numerical results are in good agreement with the experiment in terms of both mean flame shape and soot location. The flame behaviour is characterised by a strong interaction with both the turbulent structures and the evaporating spray that determine the local characteristics of the flame. The strong evaporation in the region close to the injection location causes high fluctuations of the fuel mass fraction and the small scale gradients lead to an intense mixing. The flame in this region appears lifted-off as a result of local extinction. In this region, the fuel mass fraction is relatively high and a low level of soot is observed. Further downstream, the complete pyrolysis of the fuel leads to the formation of soot precursors. Soot surface growth together with soot oxidation determine the soot mass fraction downstream of the injector exit. A strong contribution of the transport in physical space is observed for the soot mass fraction suggesting the importance of mixing on the soot formation. Results obtained in this work have shown a very promising capability of the 
LES/CMC approach to capture the main characteristics of the flame, soot formation and location at engine relevant conditions. Future work should focus on the coupling of the CMC with more advanced soot models in order to improve the quantitative prediction of the soot level.

\section{ACKNOWLEDGMENT}

The research leading to these results has received funding from Rolls-Royce plc. The experimental campaign was funded by the German Federal Ministry of Economics and Technology under contract 20T0802 ("EffMaTec"). The authors would also like to thank Ruud Eggels and Max Staufer from Rolls-Royce Deutschland for sharing the geometry of the combustor and for the very useful discussion regarding the implementation of the models in Precise-Uns.

\section{REFERENCES}

[1] Lindstedt, R., and Louloudi, S., 2005. "Joint-scalar transported PDF modeling of soot formation and oxidation". Proceedings of the Combustion Institute, 30(1), pp. 775-783.

[2] Jenny, P., Roekaerts, D., and Beishuizen, N., 2012. "Modeling of turbulent dilute spray combustion". Progress in Energy and Combustion Science, 38(6), pp. $846-887$.

[3] Pera, C., Rveillon, J., Vervisch, L., and Domingo, P., 2006. "Modeling subgrid scale mixture fraction variance in LES of evaporating spray". Combustion and Flame, 146(4), pp. 635 - 648.

[4] Olguin, H., and Gutheil, E., 2014. "Influence of evaporation on spray flamelet structures". Combustion and Flame, 161(4), pp. 987 $-996$.

[5] Triantafyllidis, A., Mastorakos, E., and Eggels, R., 2009. "Large Eddy Simulations of forced ignition of a non-premixed bluff-body methane flame with Conditional Moment Closure". Combustion and Flame, 156(12), pp. 2328 - 2345.

[6] Garmory, A., and Mastorakos, E., 2015. "Numerical simulation of oxy-fuel jet flames using unstructured LES-CMC". Proceedings of the Combustion Institute, 35(2), pp. 1207 - 1214.

[7] Zhang, H., Garmory, A., Cavaliere, D. E., and Mastorakos, E., 2015. "Large Eddy Simulation/Conditional Moment Closure modeling of swirl-stabilized non-premixed flames with local extinction”. Proceedings of the Combustion Institute, 35(2), pp. 1167 $-1174$

[8] Ukai, S., Kronenburg, A., and Stein, O., 2015. "Large eddy simulation of dilute acetone spray flames using CMC coupled with tabulated chemistry". Proceedings of the Combustion Institute, 35(2), pp. 1667 - 1674.

[9] Giusti, A., and Mastorakos, E., 2017. "Detailed chemistry LES/CMC simulation of a swirling ethanol spray flame approaching blow-off". Proceedings of the Combustion Institute, 36(2), pp. 2625-2632.

[10] Tyliszczak, A., 2015. "LES-CMC study of an excited hydrogen flame". Combustion and Flame, 162(10), pp. 3864 - 3883.

[11] Zhang, H., and Mastorakos, E., 2016. "Prediction of Global Extinction Conditions and Dynamics in Swirling Non-premixed Flames Using LES/CMC Modelling”. Flow, Turbulence and Combustion, 96(4), pp. 863-889.

[12] Giusti, A., and Mastorakos, E., 2016. "Numerical investigation into the blow-off behaviour of swirling spray flames using the LES/CMC approach". Proceeding of the ETMM-11.

[13] Giusti, A., Kotzagianni, M., and Mastorakos, E., 2016. "LES/CMC simulations of swirl-stabilised ethanol spray flames approaching blow-off”. Flow, Turbulence and Combustion, 97(4), pp. 1165-1184.

[14] Kronenburg, A., Bilger, R., and Kent, J., 2000. "Modeling soot formation in turbulent methane-air jet diffusion flames". Combustion and Flame, 121(1-2), pp. $24-40$.

[15] Bolla, M., Wright, Y. M., Boulouchos, K., Borghesi, G., and Mastorakos, E., 2013. "Soot Formation Modeling of n-Heptane Sprays Under Diesel Engine Conditions Using the Conditional Moment Closure Approach”. Combustion Science and Technology, 185(5), pp. 766-793.

[16] Schneider, D., Meier, U., Quade, W., Koopman, J., Aumeier, T., Langfeld, A., Behrendt, T., Hassa, C., and Rackwitz, L., 2010. “A new test rig for laser optical investigations of lean jet burners". Proceedings of the 27th International Congress of the Aeronautical Sciences.

[17] Freitag, S., Behrendt, T., Heinze, J., Lange, L., Meier, U., Rackwitz, L., and Hassa, C., 2011. "Study of an Airblast Atomizer Spray in a Lean Burn Aero-Engine Model Combustor at Engine Conditions". Proceedings of the ILASS-Europe 2011.

[18] Meier, U., Lange, L., Heinze, J., Hassa, C., Sadig, S., and Luff, D., 2014. "Optical methods for studies of self-excited oscillations and the effect of dampers in a high pressure single sector combustor". Proceedings of the ASME Turbo Expo(GT2014-25873). 
[19] Heinze, J., Meier, U., Behrendt, T., Willert, C., Geigle, K., Lammel, O., and Lckerath, R., 2011. "PLIF thermometry based on measurements of absolute concentrations of the OH radical". Z. Phys. Chem, 225, pp. 1315-1341.

[20] Klimenko, A., and Bilger, R., 1999. "Conditional moment closure for turbulent combustion". Progress in Energy and Combustion Science, 25(6), pp. $595-687$.

[21] Mortensen, M., and Bilger, R. W., 2009. "Derivation of the conditional moment closure equations for spray combustion". Combustion and Flame, 156(1), pp. $62-72$.

[22] Anand, M. S., Eggels, R., Staufer, M., Zedda, M., and Zhu, J., 2013. “An advanced unstructured-grid finite-volume design system for gas turbine combustion analysis". Proceedings of the ASME Gas Turbine India Conference(GTINDIA2013-3537).

[23] Tyliszczak, A., Cavaliere, D. E., and Mastorakos, E., 2014. "LES/CMC of Blow-off in a Liquid Fueled Swirl Burner". Flow, Turbulence and Combustion, 92(1), pp. 237-267.

[24] Triantafyllidis, A., and Mastorakos, E., 2010. "Implementation Issues of the Conditional Moment Closure Model in Large Eddy Simulations". Flow, Turbulence and Combustion, 84(3), pp. 481-512.

[25] Garmory, A., and Mastorakos, E., 2011. "Capturing localised extinction in Sandia Flame F with LES-CMC". Proceedings of the Combustion Institute, 33(1), pp. 1673 - 1680.

[26] Donde, P., Raman, V., Mueller, M. E., and Pitsch, H., 2013. "LES/PDF based modeling of soot-turbulence interactions in turbulent flames". Proceedings of the Combustion Institute, 34(1), pp. 1183 - 1192.

[27] Lignell, D. O., Chen, J. H., and Smith, P. J., 2008. "Three-dimensional direct numerical simulation of soot formation and transport in a temporally evolving nonpremixed ethylene jet flame". Combustion and Flame, 155(1-2), pp. 316 - 333.

[28] Nehse, M., Warnatz, J., and Chevalier, C., 1996. "Kinetic Modeling of the Oxidation of Large Aliphatic Hydrocarbons". Proceeding of the Combustion Institute, 26, pp. 773-780.

[29] Leung, K., Lindstedt, R., and Jones, W., 1991. "A simplified reaction mechanism for soot formation in nonpremixed flames". Combustion and Flame, 87(3), pp. $289-305$.

[30] Slavinskaya, N. A., Riedel, U., Dworkin, S. B., and Thomson, M. J., 2012. "Detailed numerical modeling of PAH formation and growth in non-premixed ethylene and ethane flames". Combustion and Flame, 159(3), pp. 979-995.

[31] Gepperth, S., Koch, R., and Bauer, H.-J., 2013. "Analysis and comparison of primary droplet characteristics in the near field of a prefilming airblast atomizer". Proceedings of the ASME Turbo Expo(GT2013-94033).

[32] Abramzon, B., and Sirignano, W., 1989. "Droplet vaporization model for spray combustion calculations". International Journal of Heat and Mass Transfer, 32(9), pp. 1605 - 1618.

[33] Schmehl, R., Maier, G., and Wittig, S., 2000. "CFD Analysis of Fuel Atomization, Secondary Droplet Breakup and Spray Dispersion in the Premix Duct of a LPP Combustor". Proceedings of the ICLASS.

[34] De, S., and Kim, S. H., 2013. "Large eddy simulation of dilute reacting sprays: Droplet evaporation and scalar mixing”. Combustion and Flame, 160(10), pp. 2048 - 2066.

[35] Langella, I., and Swaminathan, N., 2016. "Unstrained and strained flamelets for LES of premixed combustion". Combustion Theory and Modelling, 20(3), pp. 410-440.

[36] Sidey, J. A., Giusti, A., and Mastorakos, E., 2016. "Simulations of laminar non-premixed flames of kerosene with hot combustion products as oxidiser". Combustion Theory and Modelling, 120(5), pp. 958-973. 


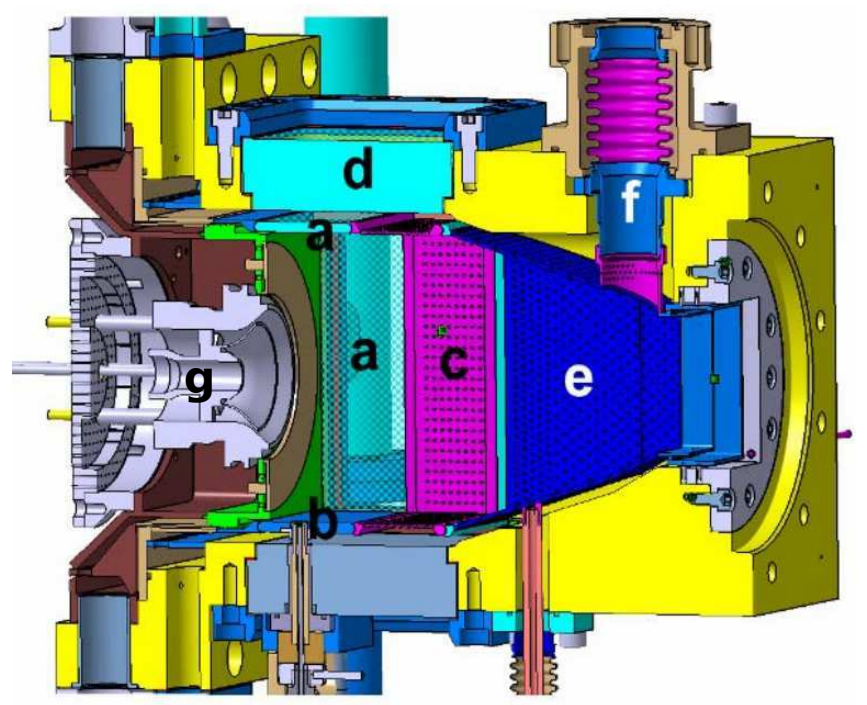

FIGURE 1. Schematic of the BOSS rig [17].

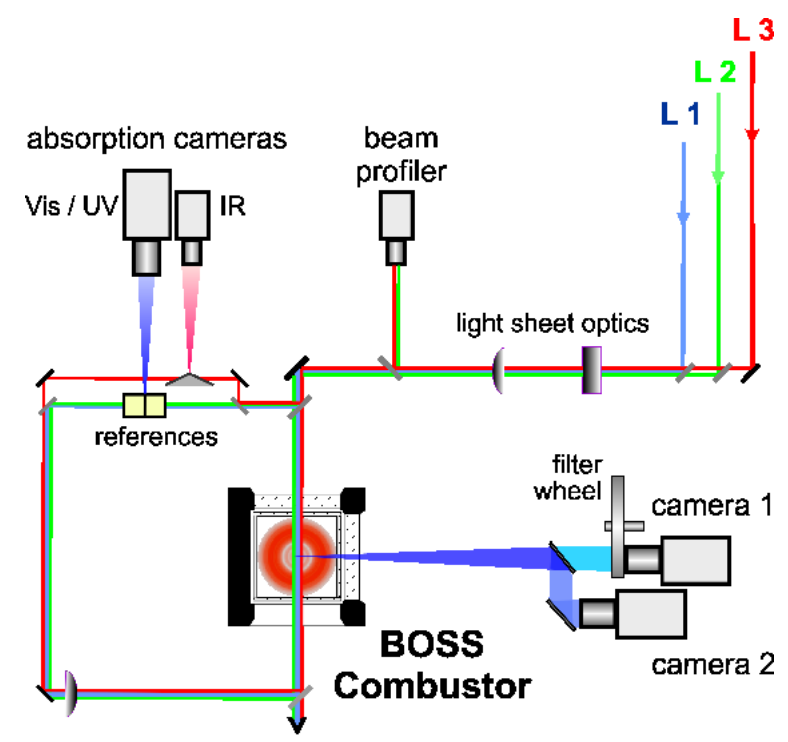

FIGURE 2. Optical setup for OH-PLIF and LII. 


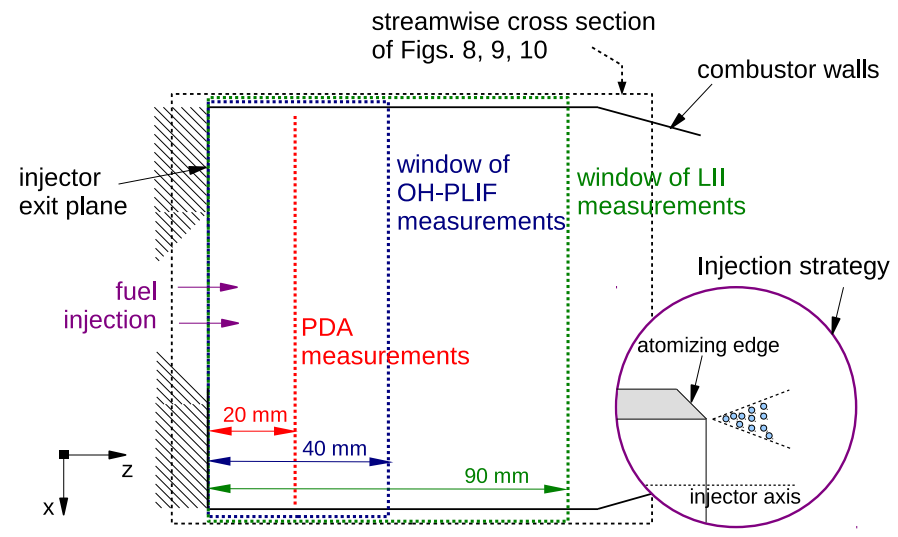

FIGURE 3. Schematic of the measurement regions for the various experimental techniques used in this work; a schematic of the injection strategy is included.

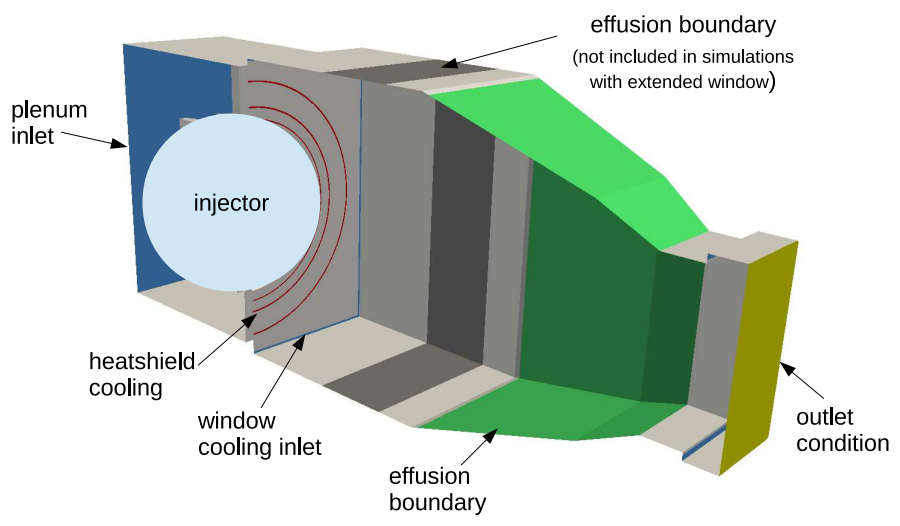

FIGURE 4. Schematic of the computational domain with relevant boundary conditions.

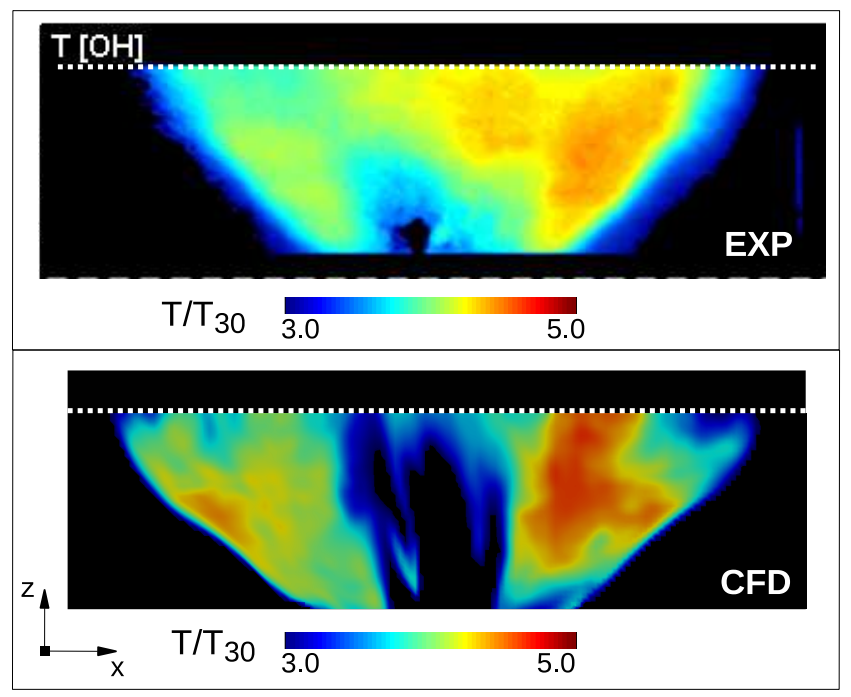

FIGURE 5. Mean temperature field from the experiment and the numerical simulation. 


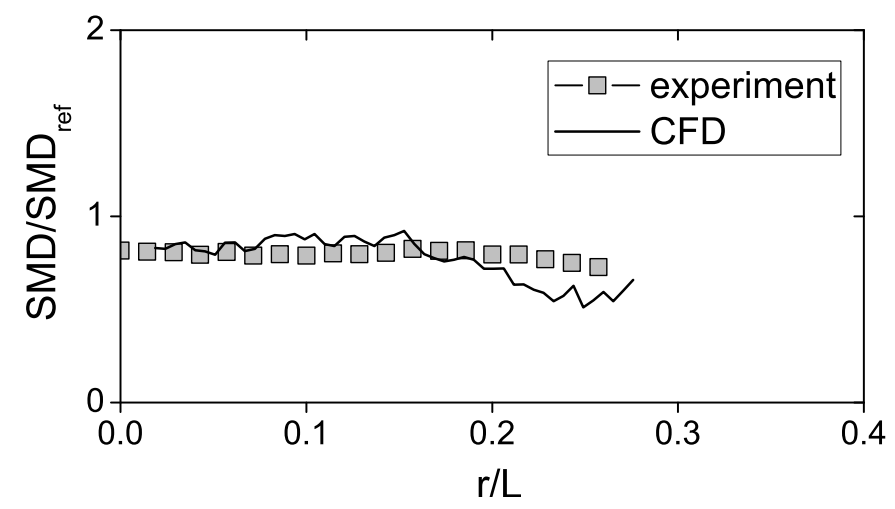

FIGURE 6. Comparison between experiment and numerical simulation in terms of SMD at a location downstream of the injector exit ( $\mathrm{z}=20 \mathrm{~mm})$ as a function of the non-dimensional radius $\mathrm{r} / \mathrm{L}$ ( $\mathrm{L}$ is the side of the square section enclosure).

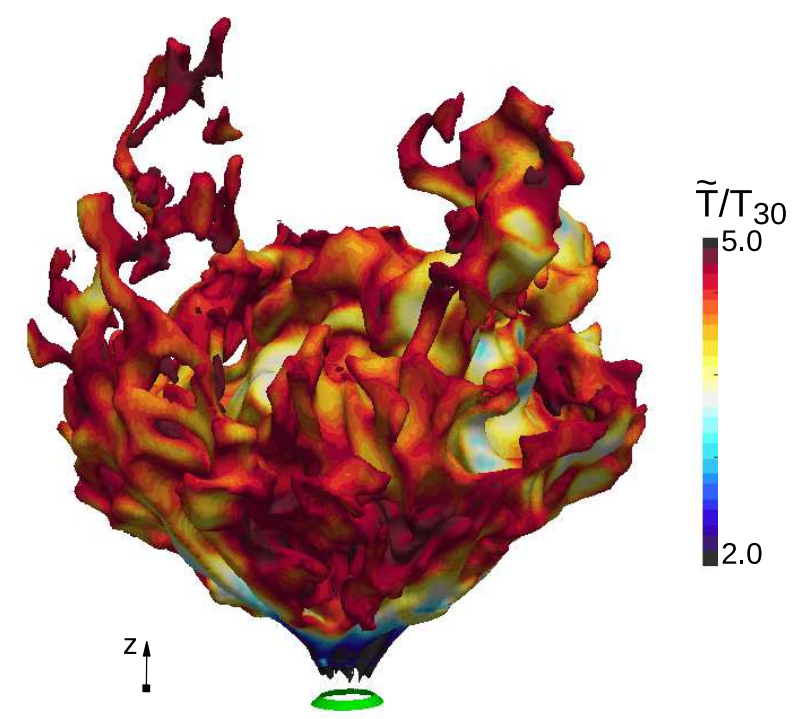

FIGURE 7. Isosurface of the stoichiometric mixture fraction coloured with temperature. 


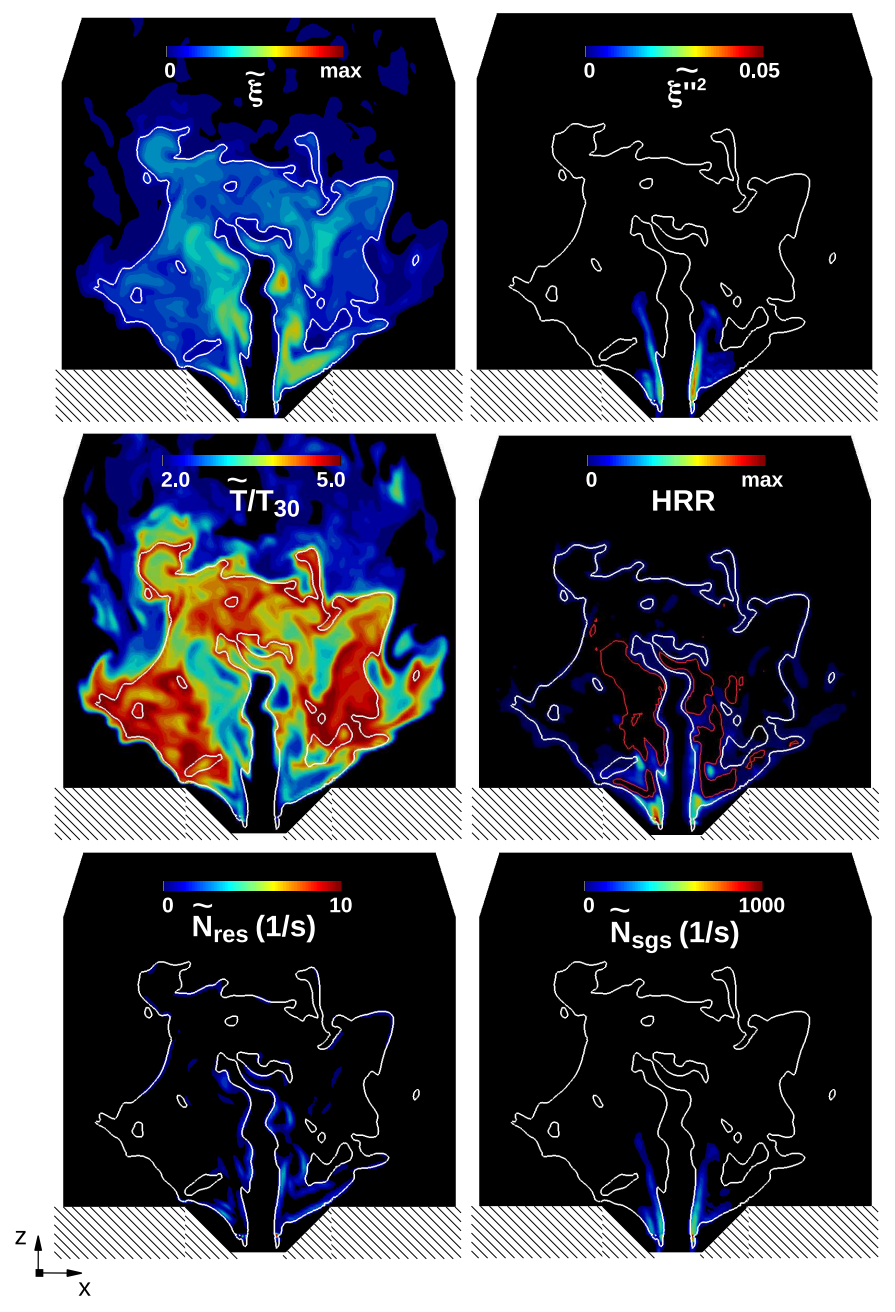

FIGURE 8. Selected instantaneous flow field quantities in a streamwise cross-section of the combustor; the white line represents the stoichiometric mixture fraction. 


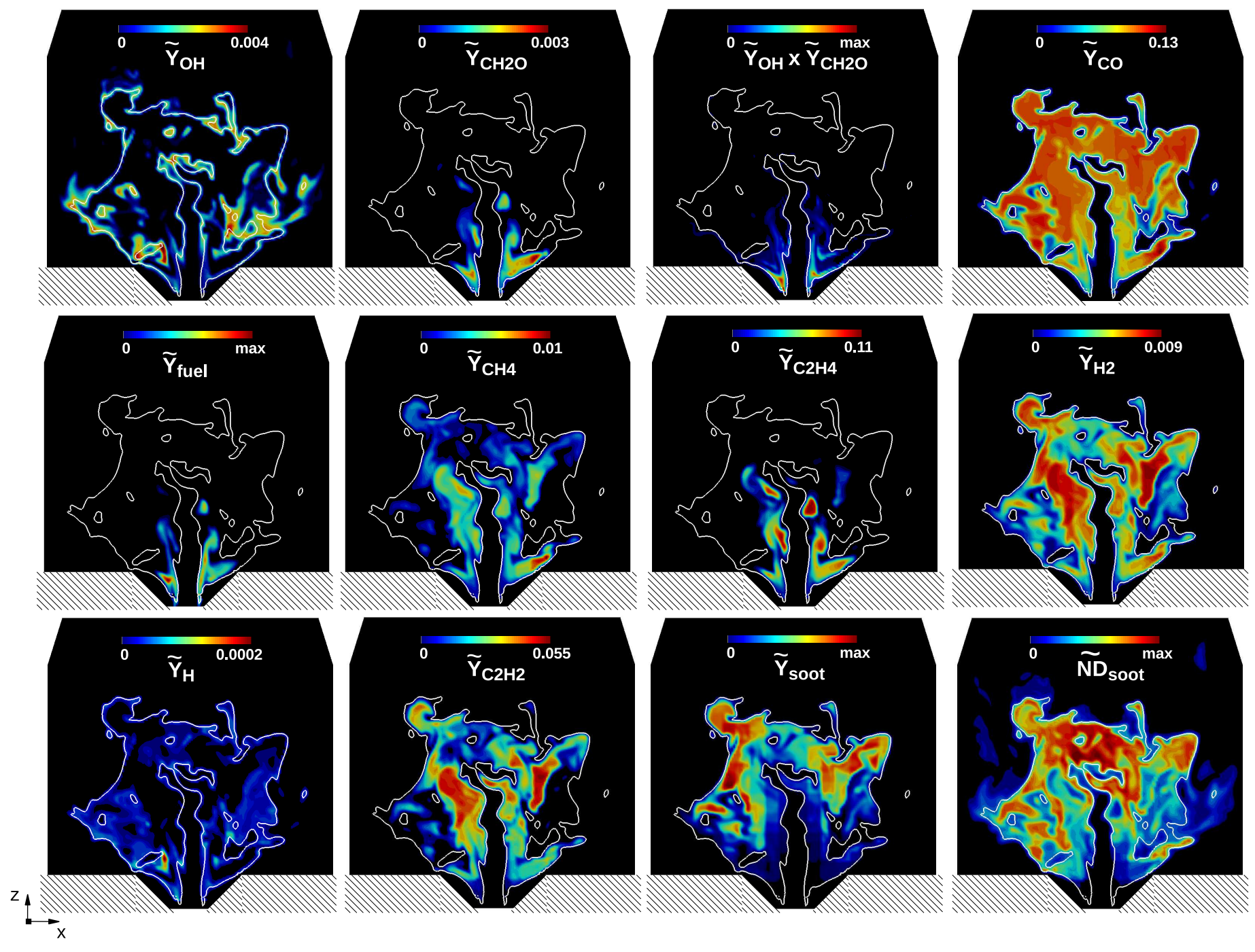

FIGURE 9. Instantaneous mass fraction, $\widetilde{Y}$, of selected species and soot number density, $\widetilde{N D}_{\text {soot }}$, in a streamwise cross-section of the combustor; the white line represents the stoichiometric mixture fraction. 


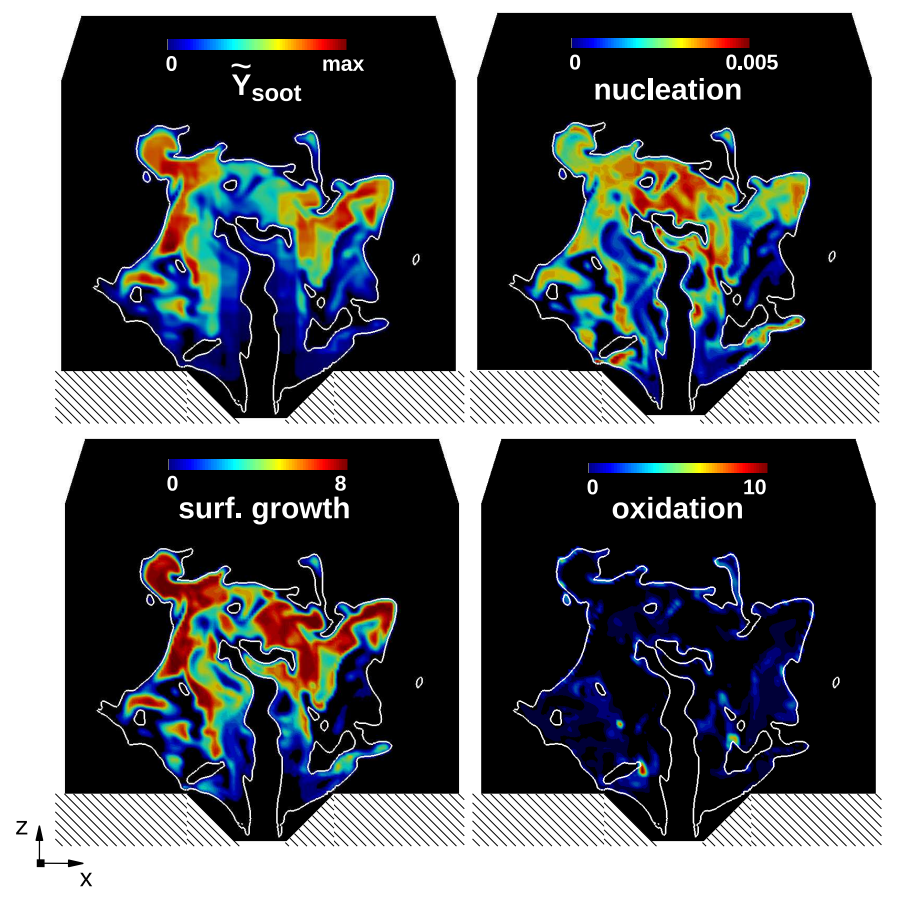

FIGURE 10. Soot nucleation, surface growth and oxidation source terms in a streamwise cross-section of the combustor; the white line represents the stoichiometric mixture fraction. 

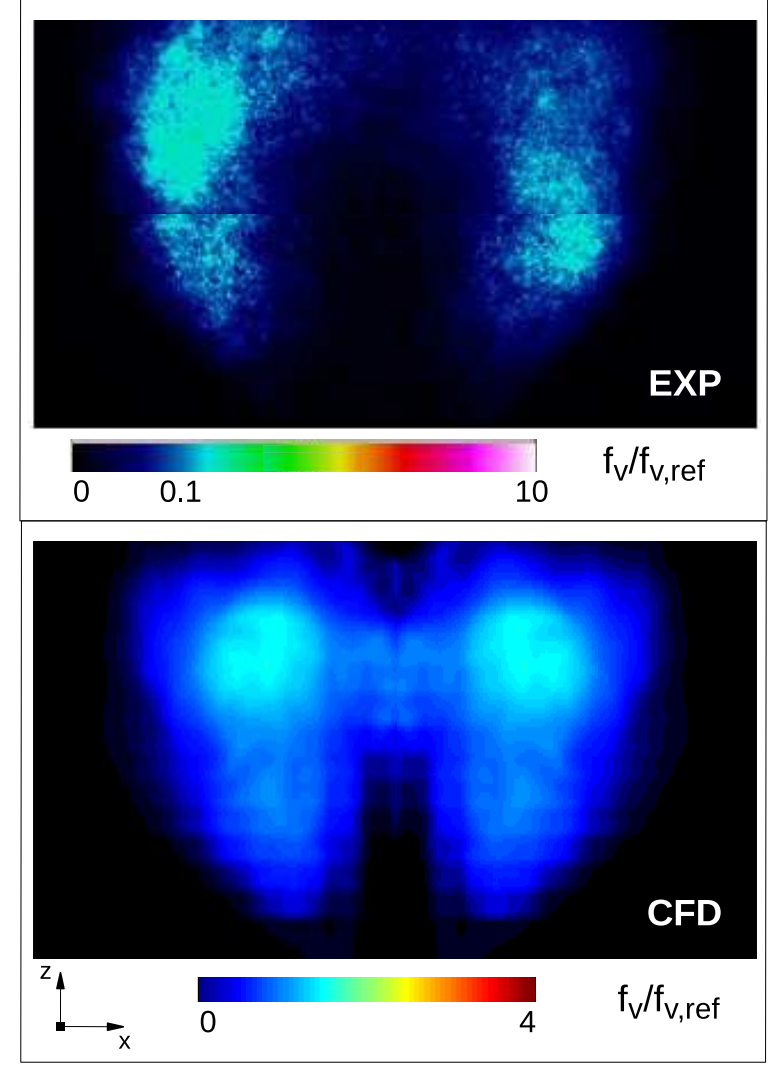

FIGURE 11. Mean soot volume fraction in the experiment and numerical simulation. 